For reprint orders, please contact: reprints@futuremedicine.com

\title{
A state-of-the-art review and guidelines for tumor treating fields treatment planning and patient follow-up in glioblastoma
}

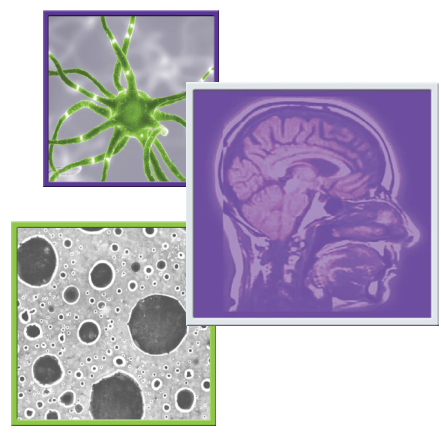

John Trusheim*, ${ }^{*}$, Erin Dunbar², James Battiste ${ }^{3}$, Fabio Iwamoto $^{4}$, Nimish Mohile ${ }^{5}$, Denise Damek ${ }^{6}$, Daniela A Bota7 \& Jennifer Connelly ${ }^{8}$

Practice Points

- Tumor treating fields (TTFields) in combination with temozolomide are a standard treatment option in patients newly diagnosed with glioblastoma $(\mathrm{GB})$ following maximal surgical debulking and completion of concurrent chemoradiation.

- Treatment planning using the NovoTAL software optimizes TTFields intensity delivered in two orthogonal directions to the gross tumor volume and the proximal peritumoral brain zone.

- Sequential neuroimaging should be reviewed in order to assess the extent of active, residual enhancing and nonmeasurable tumor when performing treatment planning.

- Treatment should be planned mapping closely to the boundaries of active tumor or to the margins of a resection cavity visualized in axial and coronal planes using T1 postcontrast or T2/fluid-attenuated inversion recovery sequences as appropriate.

- Changes in neuroimaging can occur following the initiation of treatment with TTFields. Response assessment should incorporate a comprehensive evaluation of the patient's clinical status, concurrent therapies received, treatment compliance and general scalp health when assessing clinical response.

- The median duration of TTFields therapy in a pivotal Phase III study conducted in newly diagnosed GB patients was 9 months, with patients remaining on TTFields with a change to second-line chemotherapy through first progression.

- Treatment can be re-planned if there are significant changes in imaging from baseline.

- The development of TTFields treatment planning and response assessment algorithms aim to help standardize patient care across specialties and institutions caring for patients with GB.

Tumor treating fields (TTFields) are an integral treatment modality in the management of glioblastoma and extend overall survival when combined with maintenance temozolomide in newly diagnosed patients. Complexities exist regarding correct selection of imaging sequences with which to perform TTFields treatment planning. Guidelines are warranted first, to facilitate treatment planning standardization across medical disciplines and institutions, to ensure optimal TTFields delivery to the tumor and peritumoral brain zone while maximizing

'Abbott Northwestern Hospital Neuroscience Institute, Minneapolis, MN, USA

2PiedmontBrain Tumor Center, Atlanta, GA, USA

${ }^{3}$ Oklahoma University Health Sciences Center, Oklahoma City, OK, USA

${ }^{4}$ The Neurological Institute of New York, Columbia University, New York, NY, USA

5University of Rochester Medical Center, Rochester, NY, USA

${ }^{6}$ University of Colorado Hospital, Aurora, CO, USA

${ }^{7}$ University of California, Irvine Medical Center, Orange, CA, USA

${ }^{8}$ Froedtert Hospital \& The Medical College of Wisconsin, Milwaukee, WI, USA

*Author for correspondence: Tel.: +1 952920 7200; Fax: +1 612863 3732; John.Trusheim@Mpls-clinic.com

Future Medicine ${ }_{\text {part of }}$ 
patient safety, and also to mitigate the risk of premature cessation of a potentially beneficial treatment. This summary guideline outlines methods for starting patients on TTFields, for monitoring patient response to therapy and provides a framework for evaluating when therapy should be re-planned, based on the extent of sequential imaging changes.

First draft submitted: 28 July 2016; Accepted for publication: 23 August 2016; Published online: 15 September 2016

\section{KEYWORDS}

- alternating electric fields

- glioblastoma • guidelines

- NovoTAL • response assessment $\bullet$ treatment planning • TTFields • tumor treating fields

\section{Background}

Tumor treating fields (TTFields) are an integral modality in the management of patients with newly diagnosed and recurrent glioblastoma (GB), in addition to surgery, radiation and chemotherapy. TTFields are a regionally delivered treatment utilizing alternating electric fields to disrupt highly orchestrated intracellular processes necessary for successful cell division [1-5]. TTFields are clinically delivered in paired orthogonal directions, left-right and anterior-posterior, using Optune ${ }^{\mathrm{TM}}$ (NovoTTF$100 \mathrm{~A}$ System). Optune is a portable medical device consisting of a TTFields generator, custom backpack and insulated ceramic discs called transducer arrays, which are applied directly to a patient's shaved scalp (Supplementary Figure 1) $[6,7]$. The device is preset to deliver TTFields at a frequency of $200 \mathrm{kHz}$ and is operated by the patient independently. It is monitored periodically by device specialists, who are available 24/7 to provide technical support to the patient, their family and physician.

TTFields are nonionizing and are delivered at an intermediate frequency $(200 \mathrm{kHz}$ for $\mathrm{GB})$ and low intensities $(1-3 \mathrm{~V} / \mathrm{cm}$ ) leading to a variety of downstream, antimitotic biologic effects, sparing quiescent cells. These include disruption of normal mitotic spindle and cytokinetic contractile band assembly $[1,4]$, mitotic arrest [8], dielectrophoresis of charged intracellular macromolecules during cytokinesis [9,10], which ultimately result in polyploidy and aneuploidy in cellular progeny, leading to a loss of clonogenic potential [4,11,12], and immunogenic cell death [13]. When applied to GB cell lines in in vitro studies, TTFields can also induce other stress effects potentially culminating in the activation of immune-mediated and other death pathways [4,11,14]. In addition, TTFields have also been shown to induce autophagy, and can modulate cellular propensity to migrate and invade adjacent tissues $[15,16]$. Preclinical studies in GB and other cancer cell lines demonstrate that incremental cytotoxic effects can be observed when TTFields' intensity is increased from 1 to $2-3 \mathrm{~V} / \mathrm{cm}$, with complete arrest of cellular proliferation observed with field intensities exceeding $2.25 \mathrm{~V} / \mathrm{cm}[1,5]$. TTFields exert maximal effects when aligned to a cell's mitotic axis. As a cell's mitotic axis can occur randomly in any direction, additive cytotoxic effects are also observed when TTFields are applied in multiple sequential directions [5].

Although the direct measurements of TTFields intensities delivered to the brain are not performed routinely, advanced electric field simulation studies can provide insights into the behavior and distribution of TTFields in the clinical setting. Spatial distributions of electric fields within the brain parenchyma have been determined in realistic head models computed with finite element method (FEM) simulations $[17,18]$. Image segmentation initially separates major tissue types such as scalp, skull and gray and white matter using MRI sequences and then model transducer arrays are positioned directly on the scalp [18]. The dimensions and separation of model electrodes accurately represent the geometry of ceramic discs used clinically. Volume meshes are generated and solved using the FEM framework with preset electric field parameters of $200 \mathrm{kHz}$, and isotropic tissue conductivity and relative permittivity values. A corresponding electromagnetic wavelength for a propagating field at a similar frequency would be on the order of $1 \mathrm{~km}$. As TTFields are applied across the head at much shorter distances $(\sim 20-30 \mathrm{~cm})$, it is not possible to precisely focus the field to target discrete structures, as is possible with radiosurgery. Instead, TTFields will distribute nonuniformly mainly throughout the supratentorial brain. Electric field intensities can differ significantly based on the heterogeneous dielectric tissue properties of cranial structures such as the gray matter, white matter tracts and cerebrospinal fluid [17-19], tumor location, tumor properties and transducer array layout [20]. As the majority of GB will recur at the margin of a resection cavity or within the proximal $20 \mathrm{~mm}$ 
of peritumoral brain zone (PBZ) to the original tumor bed [21-23], it is important to understand the distribution of TTFields in these regions. Recent simulation studies have shown that electric field intensity delivered to the gross tumor volume (GTV) and proximal PBZ exceeds therapeutic thresholds of $1 \mathrm{~V} / \mathrm{cm}$ in both orthogonal directions for models with solid brain tumors (Supplementary Figure 2) [24].

In clinical practice, TTFields are indicated in combination with maintenance temozolomide (TMZ) for the treatment of patients with newly diagnosed GB, and as monotherapy for patients with recurrent disease [25,26]. TTFields gained US FDA approval for newly diagnosed GB patients based on the positive results of the Phase III randomized controlled, EF-14 study [25]. At the first prespecified interim analysis, this trial demonstrated that 315 patients with newly diagnosed GB (who had undergone maximal surgical debulking followed by six cycles of concurrent chemoradiation), randomized 2:1 to receive TTFields with maintenance TMZ, experienced a significantly longer median progression-free survival (PFS) and median overall survival (OS) compared with patients receiving maintenance TMZ alone (20.5 vs 15.6 months, hazard ratio [HR]: $0.64 ; \mathrm{p}=0.004)$. The addition of TTFields to standard of care did not increase serious adverse events and did not impair performance status or quality of life [27]. In the EF-14 study, patients randomized to the TTFields/TMZ treatment arm could remain on TTFields at first progression and switch to second-line chemotherapy [25]. In a post hoc analysis, OS was significantly higher from first progression, in patients receiving TTFields in combination with second-line chemotherapy compared with patients receiving second-line chemotherapy alone (11.8 vs 9.2 months, HR: $0.695 ; \mathrm{p}=0.0489)$ [28]. TTFields were originally approved in the recurrent GB setting as monotherapy, based on the results of the randomized controlled Phase III, EF-11 study [26]. This randomized trial demonstrated equivalent efficacy (median OS: 6.6 vs 6.0 months; HR: $0.86 ; p=0.27$ ) combined with improved safety and quality of life in patients receiving TTFields monotherapy compared with patients receiving best physician's choice of chemotherapy [26]. As TTFields are a locally directed therapy with no half-life, systemic side effects observed with chemotherapy are not typically seen in patients receiving TTFields monotherapy. The most common treatment-related adverse events are dermatologic in nature and have been reviewed in detail previously [29]. Skin irritation can occur beneath the transducer arrays as a consequence of chemical irritation from the hydrogel, local moisture at the skin surface or due to allergic irritation in response to the hydrogel and adhesive tape. In addition, as treatment requires shaving of the scalp and replacing the transducer arrays two- to three-times per week, mechanical trauma from repeat shaving and physical stresses from removal and reapplication of the arrays can also predispose the skin to erosions. Pressure from the ceramic transducer discs can lead to decreased skin perfusion, a risk that is exacerbated in the presence of scars, hardware and in patients who have received prior radiation therapy. In some cases, this can lead to the development of an ulcer. Inappropriate scalp care can also lead to skin infections and folliculitis. The majority of dermatologic adverse events are mild to moderate in nature and can be managed with high-potency topical steroids or topical antibiotics without requiring breaks in treatment. Grade 3/4 adverse events were observed in $2 \%$ of patients receiving TTFields monotherapy in the EF-11 trial [26]. The TTFields device is contraindicated in patients with active implanted electronic medical devices such as deep brain stimulators, pacemakers and programmable shunts, and in patients with skull defects such as a missing bone flap, due to the risk of skin toxicity and tissue damage. It should also not be used in patients with known hypersensitivity to conductive hydrogels or in patients with infratentorial disease.

In order to maximize the intensity of electric field delivered at the site of active tumor, treatment is individualized for each patient using the Novocure Transducer Array Layout - NovoTAL ${ }^{\mathrm{TM}}$ System (NovoTAL, USA) [30]. In simulation studies, personalizing treatment results in near doubling of TTFields intensity delivered to the GTV compared with TTFields delivery using a default symmetric array layout [20]. Treatment planning with NovoTAL is required for any patient commencing treatment with TTFields. In the clinical trial setting, treatment planning was historically performed by the study sponsor's clinical team using the patient's baseline MRI results. In the clinical practice setting, physicians certified to prescribe TTFields who are managing patients with GB, may elect to perform their own treatment planning by 
completing a NovoTAL certification program, presently only available in the USA. This program provides education on the fundamental principles of electric field distribution theory and practical training on obtaining head morphometric measurements, as well as tumor coordinates from MRI scans. A basic requirement for this certification is that physicians have familiarity with reading and interpreting neuroimaging. As the treating physician may have comprehensive information regarding the patient's clinical history, current clinical status and knowledge of sequential imaging changes, there, may be potential benefits for the patient(s) in having their treating physician plan therapy directly.

\section{- Overview of TTFields treatment planning using NovoTAL}

Conventional treatment mapping using the FDA-approved NovoTAL software [30] is performed by planning treatment to the extent of contrast-enhancing disease most indicative of active tumor (methods described previously) [3033]. A measure of axial head size is first obtained using the image at the uppermost limit of the orbit on a T1-weighted sequence (Figure 1A). A fiducial frame is placed around the head bordering the outer margin of the scalp on four sides. Measurements for anteroposterior (A-P), rightto-left $(\mathrm{R}-\mathrm{L})$ and right to anatomic midline distances are obtained from the level of the scalp, extending tangentially from a right-anterior marker origin. Coronal head size is assessed in a similar manner, using a box drawn around the head to the lower margin of cerebrum on an MRI image at the level of the external auditory canal (Figure 1B). Conventional tumor location measurements are obtained from T1-weighted postcontrast sequences in axial and coronal views, selecting images that contain the maximal diameter of contrast enhancement. Boxes are drawn around the head commencing at the outer margin of the scalp axially and encompassing the extent of supratentorial cerebrum coronally. The same measurements described previously (anteroposterior, $\mathrm{R}-\mathrm{L}$ and right to midline) are obtained (Figure $1 C \& D$ ). In addition, four measures for tumor coordinates are obtained commencing from the reference frame and from a right-anterior or right-superior origin, respectively. On axial views, these are measurements from the right reference frame to the near tumor margin and to the far tumor margin, and the distance from the front of the reference frame to the near tumor margin and to the far tumor margin. On the coronal slice capturing the maximal diameter of contrast enhancement, the four tumor coordinates are measured from the right reference frame to near tumor margin and to the far tumor margin, and from the top of the reference frame to the near tumor margin and to the far tumor margin. All measurements are rounded to the nearest millimeter and are entered sequentially into the NovoTAL software. Based on the data derived from FEM simulations, the software algorithm will calculate the optimal transducer array layout, which will maximize TTFields intensity at the site of the tumor based on the patient's cranial morphometry.

Although the process described above is based on MRI measurements obtained from postcontrast imaging, in the clinical setting gliomas can be very heterogeneous in nature, and thus the extent of active disease may not exclusively be captured in postcontrast sequences [34-36]. GBs may present with non-CE (NCE) T2-weighted/ fluidattenuated inversion recovery (FLAIR) signal, or more commonly may be represented in a combination in contrast-enhancing and NCE frames. A patient will minimally require an MRI with T1-weighted, T2-weighted/FLAIR and gadolinium-enhanced sequences in axial and coronal planes for treatment planning. A combination of these sequences can be used for planning purposes, as deemed appropriate by the treating physician. Although mapping based on discrete regions of contrast enhancement may result in a more focal planning approach, incorporation of margins of macroscopically normal brain or of wider regions of T2/FLAIR abnormality can alter the intensity of TTFields in areas where GB most commonly recurs such as the GTV, tumor margin and PBZ [21]. Maximizing field intensity within these zones could prove to be crucial for prolonging PFS and for improving patient outcomes overall. The PBZ, which macroscopically and radiologically resembles normal tissue, frequently harbors infiltrating tumor cells, tumorigenic stromal cells and proinflammatory cells, which collectively support local tumor recurrence [22]. As such, a physician may theoretically wish to include a wider margin on NCE tissue into the treatment plan for TTFields in order to treat residual infiltrating tumor cells. Results of treatment planning simulation studies exploring the inclusion of a margin of macroscopically normal tissue around 

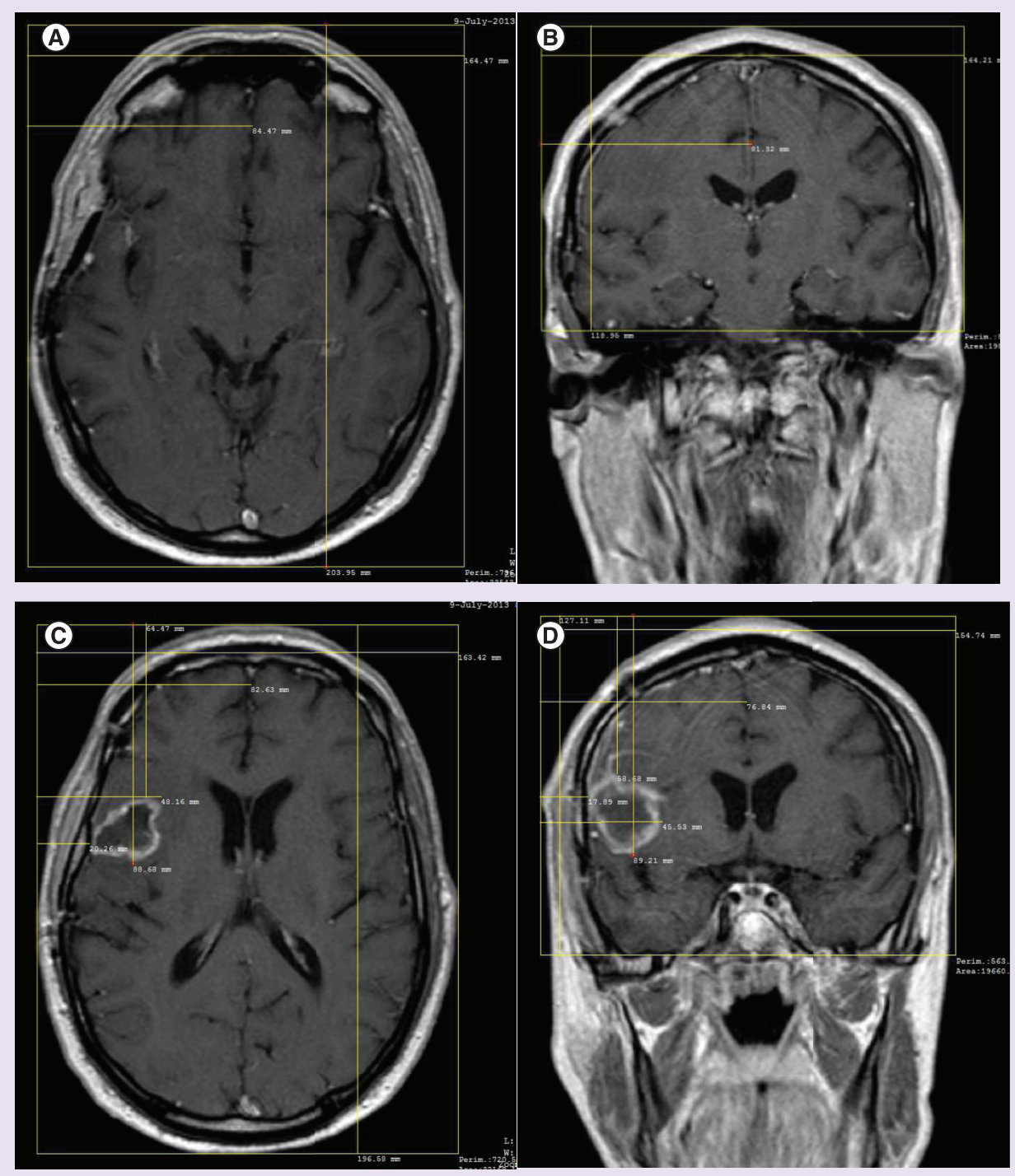

Figure 1. Standard T1-weighted postcontrast MRI measurements required to perform NovoTAL treatment planning. (A) An axial slice at the apical level of the orbit. A reference frame is drawn at the level of the scalp, and measurements for anteroposterior, left-right and right-to-midline are drawn. (B) A coronal slice at the level of the external auditory canal used to estimate the extent of cerebrum. A reference frame is drawn from the level of the scalp to an inferior boundary at the lower extent of temporal lobe, and measurements for right-left, right-to-midline and superiorto-inferior boundary are obtained. (C) Axial tumor coordinates drawn on a slice demonstrating the maximal tumor diameter. The same frame and initial measurements are repeated. In addition, measurements from the right frame to near and far tumor margins, as well as the anterior frame to near and far tumor margins, are added. (D) Coronal tumor coordinates drawn on a slice depicting the maximal diameter of tumor. The lower boundary of the box is drawn at the lowest level of visible supratentorial brain. The three initial head size measurements are repeated. In addition, measurements from the right frame to the near and far tumor margins, and from the top of the frame to the near and far tumor margin are obtained.

a boundary of contrast-enhancing tumor suggest that field intensity is optimized in the GTV and PBZ, when treatment is planned mapping to the border of contrast-enhancing disease, as opposed to mapping to include wider margins of NCE PBZ. As such, this guideline recommends 
planning therapy as closely as possible to the margins of areas representing active tumor.

\section{- Treatment planning for newly diagnosed GB}

In the newly diagnosed setting, an MRI performed approximately 4 (range: 2-6) weeks following completion of chemoradiation should be used for TTFields treatment planning purposes. However, supplemental information from the preoperative, postoperative and preradiation therapy scans (when available) can also help inform the region most likely to represent residual active tumor and target any microscopic disease. The minimum MRI sequences required are precontrast T1, T2/FLAIR and postcontrast T1 (axial and coronal), and the recommended slice thickness is $\leq 5 \mathrm{~mm}$ with no gap. In rare instances, where a patient cannot undergo MRI, a CT scan with contrast can be used for treatment planning, as long as axial and coronal head size and tumor position can be clearly delineated. Figure 2 describes the algorithm for treatment planning in patients with newly diagnosed with GB.

In the postradiation setting, an important consideration is to distinguish post-treatment imaging changes such as pseudoprogression $[37,38]$ from true disease progression. As mentioned previously, field intensity will be maximized in the GTV and PBZ, when the field is mapped as closely as possible to the margin of active tumor, so care should be taken not to overestimate the extent of active residual tumor. As reactive

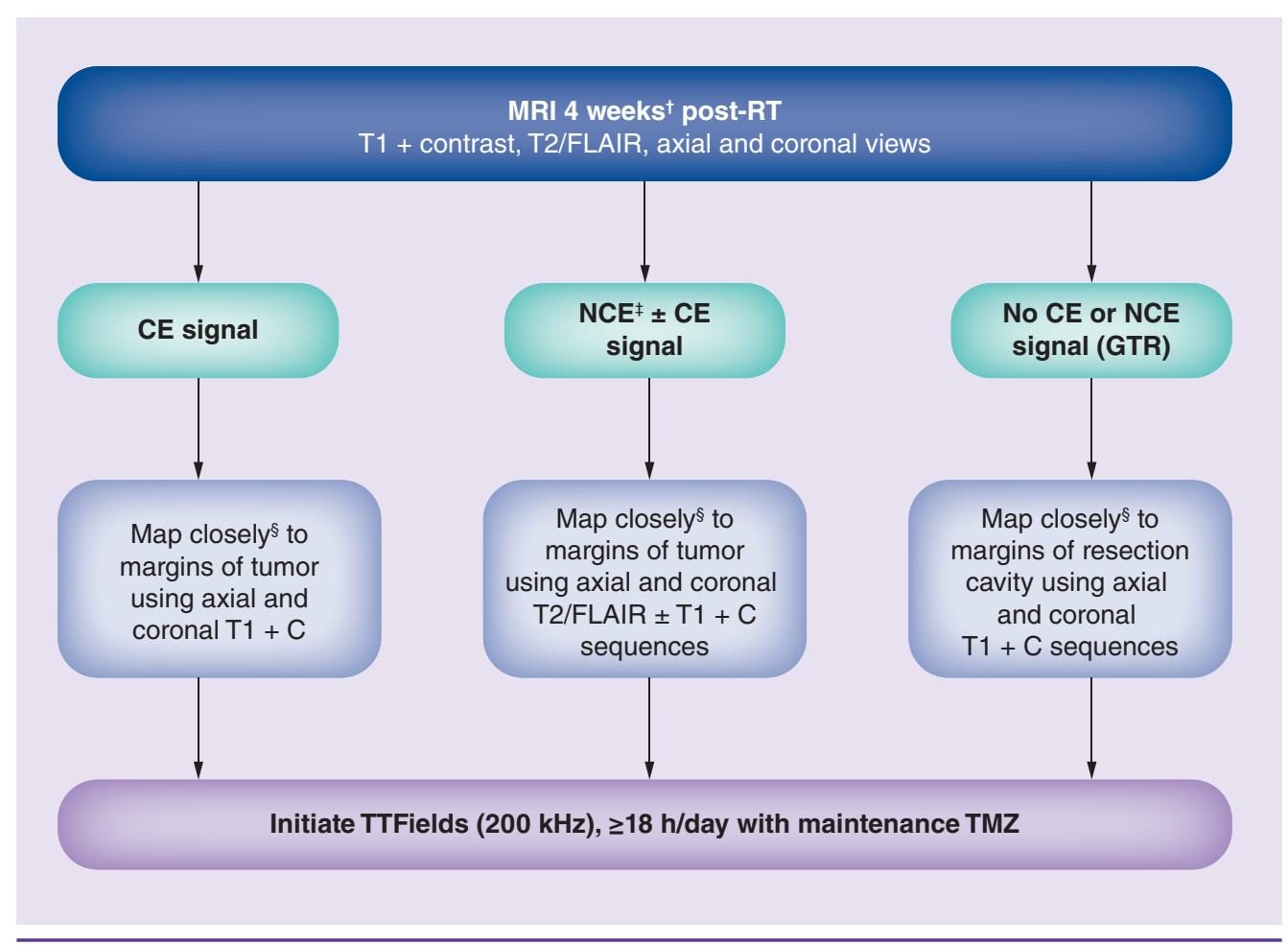

Figure 2. Tumor treating fields treatment planning algorithm for newly diagnosed glioblastoma. ${ }^{\dagger} \mathrm{MRI}$ obtained 2-6 weeks following completion of concurrent chemoradiation is recommended for treatment planning in patients with newly diagnosed glioblastoma. Where available, compare preoperative and pre-RT sequences to determine areas of active disease versus post-treatment sequelae.

"In instances where a newly diagnosed patient has exclusively NCE signal, mapping should be performed using axial and coronal T2/FLAIR images, closely approximating to the margins of the tumor. ${ }^{8}$ Coordinates for tumor location should closely approximate the edge of the region most representative of active tumor without including extra margin of normal tissue. This approach will maximize the field intensity in both the tumor volume and proximal peritumoral brain zone.

C: Contrast; CE: Contrast enhancement; FLAIR: Fluid-attenuated inversion recovery; GTR: Gross total resection; NCE: Noncontrast enhancement; RT: Radiotherapy; TMZ: Temozolomide. 

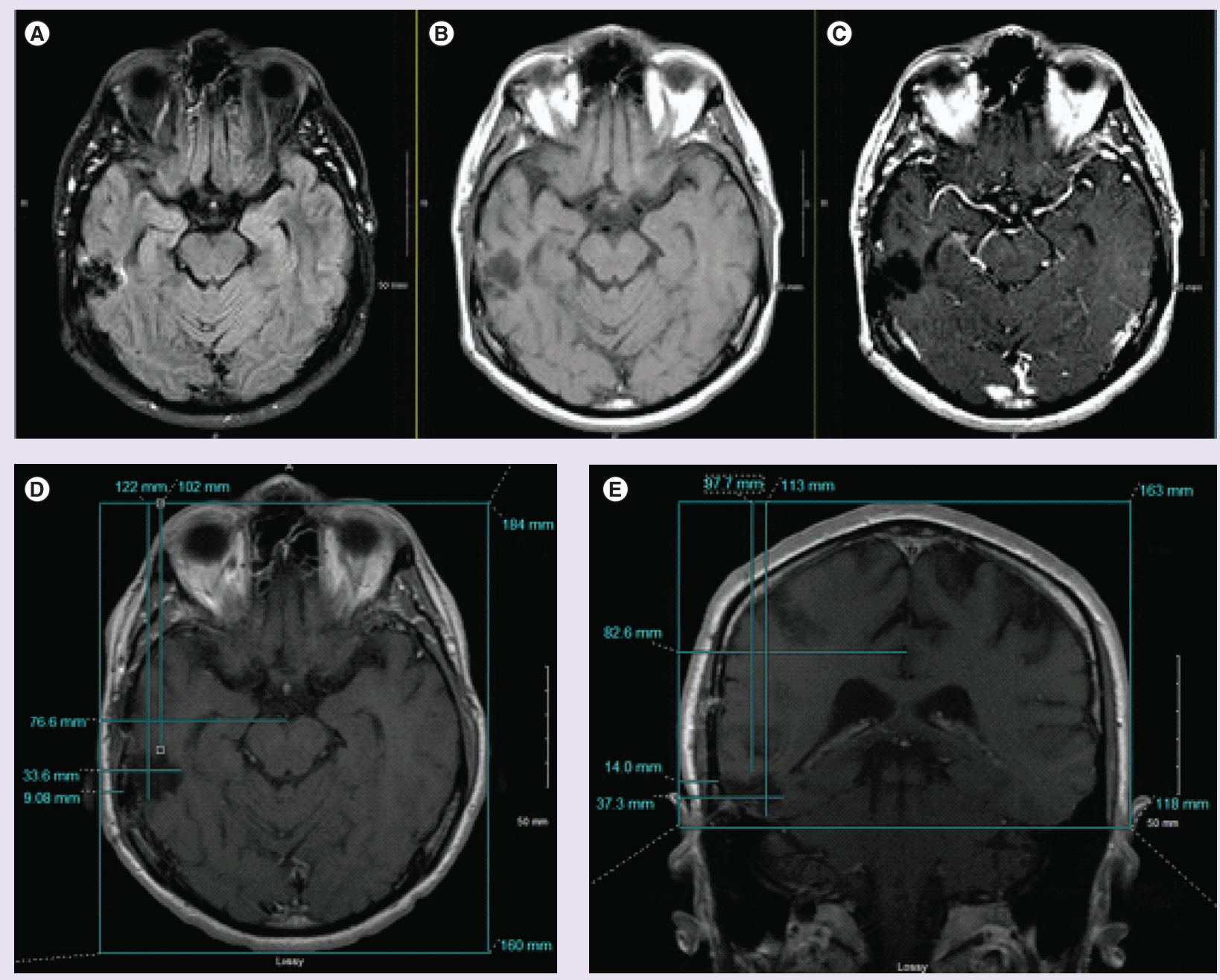

Figure 3. Treatment planning in patient post gross total resection with no evidence of residual disease. (A-C) (from top left to right) Post-radiotherapy axial views on fluid-attenuated inversion recovery, $\mathrm{T} 1$ and $\mathrm{T} 1$ with contrast sequences for a patient post-gross total resection. Head size measurements are obtained as standard on slices at the apical level of the orbit on axial views and at the level of the auditory canal on coronal views (not shown). (D \& E) Treatment planning to the boundaries of the resection cavity on axial and coronal views, respectively. On axial views, the reference frame is drawn around the head, excluding the nose anteriorly. On coronal slices, the reference frame is drawn at the level of the scalp, excluding the ears, and the inferior margin is drawn to the lowest level of visible tentorium.

alterations in the blood-brain barrier with contrast enhancement may mimic tumor progression, there may be clinical value in obtaining additional imaging such as a magnetic resonance perfusion or PET scan in order to determine which areas represent active tumor [39-41]. For patients who have undergone a gross total resection and have neither residual contrast-enhancing nor NCE signals, treatment planning should be performed mapping as closely to the margins of the resection cavity as possible (Figure 3). The output from the NovoTAL software - the transducer array layout map - should subsequently be evaluated in the context of any surgical scars, impaired skin or palpable hardware that may impact the application of the transducer arrays on the patient's scalp. TTFields are contraindicated in patients with skull defects where the bone was not replaced and the arrays should not be placed directly above any palpable hardware, screws or underlying serious skin condition. A slight shifting of the arrays is appropriate in such instances.

In patients who have undergone biopsy or subtotal resection (those with residual tumor), sequential scans should be reviewed to determine 
the areas that represent most active areas of tumor. Mapping should be performed using the postcontrast and/or T2/FLAIR sequences as appropriate. The size of the planning field should conform to the boundary of active tumor, avoiding and excluding blood products or cystic cavities not surrounded by active tumor, in order to optimize the field intensity in the tumor volume and PBZ.

\section{- Treatment planning for recurrent GB}

There are additional practical considerations when planning treatment with TTFields for patients with recurrent $\mathrm{GB}$, again with regard to evaluating and interpreting imaging changes and assessing the effects of prior therapies. Tumor progression can often be challenging to distinguish from pseudoprogression [42] or radiation necrosis. Given the frequent use of bevacizumab in recurrent $\mathrm{GB}$, pseudoresponse should also be carefully evaluated in patients receiving antiangiogenic therapy $[37,43,44]$. These scenarios can lead to both an over- or under-estimation of active tumor volume in the context of TTFields planning and may lead to suboptimal delivery of TTFields. In this setting, additional information obtained from advanced imaging modalities such as diffusion-weighted imaging, apparent diffusion coefficient maps, magnetic resonance spectroscopy and perfusion-weighted imaging may help correlate regions that represent active tumor and distinguish tumor from pseudoprogression [45,46]. Patient's scalp health should also be carefully evaluated when considering TTFields in the recurrent GB setting. Prior exposure to systemic chemotherapies and radiation may render patients more susceptible to skin toxicity (the most common devicerelated adverse event reported with TTFields treatment); therefore, regular evaluation of the patient's scalp while receiving therapy can help prevent the development of local skin irritation beneath the transducer arrays [29]. Figure 4 describes the algorithm for treatment planning in patients with recurrent GB. The most recent MRI scan showing tumor progression should typically be used for TTFields treatment planning in patients with recurrent GB. A careful comparison between prior scans should be made in order to identify the areas of active tumor. This is especially important in patients who have received antiangiogenic therapy, in whom prior imaging (when available) should be evaluated with the most recent scan. Many patients receiving bevacizumab commonly progress with a nonenhancing signal abnormality and therefore are more likely to require the inclusion of T2/FLAIR sequences in treatment planning. The treating physician should correlate the imaging findings with the patient's clinical status and determine the region where the TTFields should be focused. The mapping coordinates should conform closely to the margin of the active tumor as this will maximize field intensity in the tumor volume and the PBZ (Figure 5).

\section{- Treatment planning for multifocal GB}

Approximately one in five patients with GB may present with multifocal or multicentric disease manifesting with assorted distinct foci of enhancing tumors [47]. TTFields are a viable modality in the treatment of such patients, as TTFields distribute at therapeutic intensities within large areas of the supratentorial brain [17]. The NovoTAL software can accommodate treatment planning for any number distinct lesions $(>1 \mathrm{~cm}$ apart) within its algorithm. Any lesions which are $<1 \mathrm{~cm}$ apart should be treated as one contiguous lesion. The approach to planning therapy in cases with multifocal disease is the same as described previously; once measurements for axial as well as coronal head size have been obtained, each lesion should be delineated and measured separately, and the coordinates entered sequentially into the NovoTAL software (Figure 6).

\section{- Response assessment \& remapping TTFields}

Once TTFields are initiated, the treating physician should monitor the patient's monthly compliance report to ensure that patient is receiving the maximal benefit from the treatment (the recommended goal is to be on therapy $>18 \mathrm{~h}$ per day), and should periodically assess the scalp so that any local skin irritation is appropriately managed. A follow-up MRI scan should routinely be performed 2-3 months following treatment start as is the standard practice for GB follow-up. There are unique challenges associated with the ongoing evaluation and interpretation of imaging changes in response to treatment with TTFields, which are compounded by observations from clinical trials suggesting that there is no definitive correlation between objective radiographic response and overall patient benefit. Post hoc analyses have 


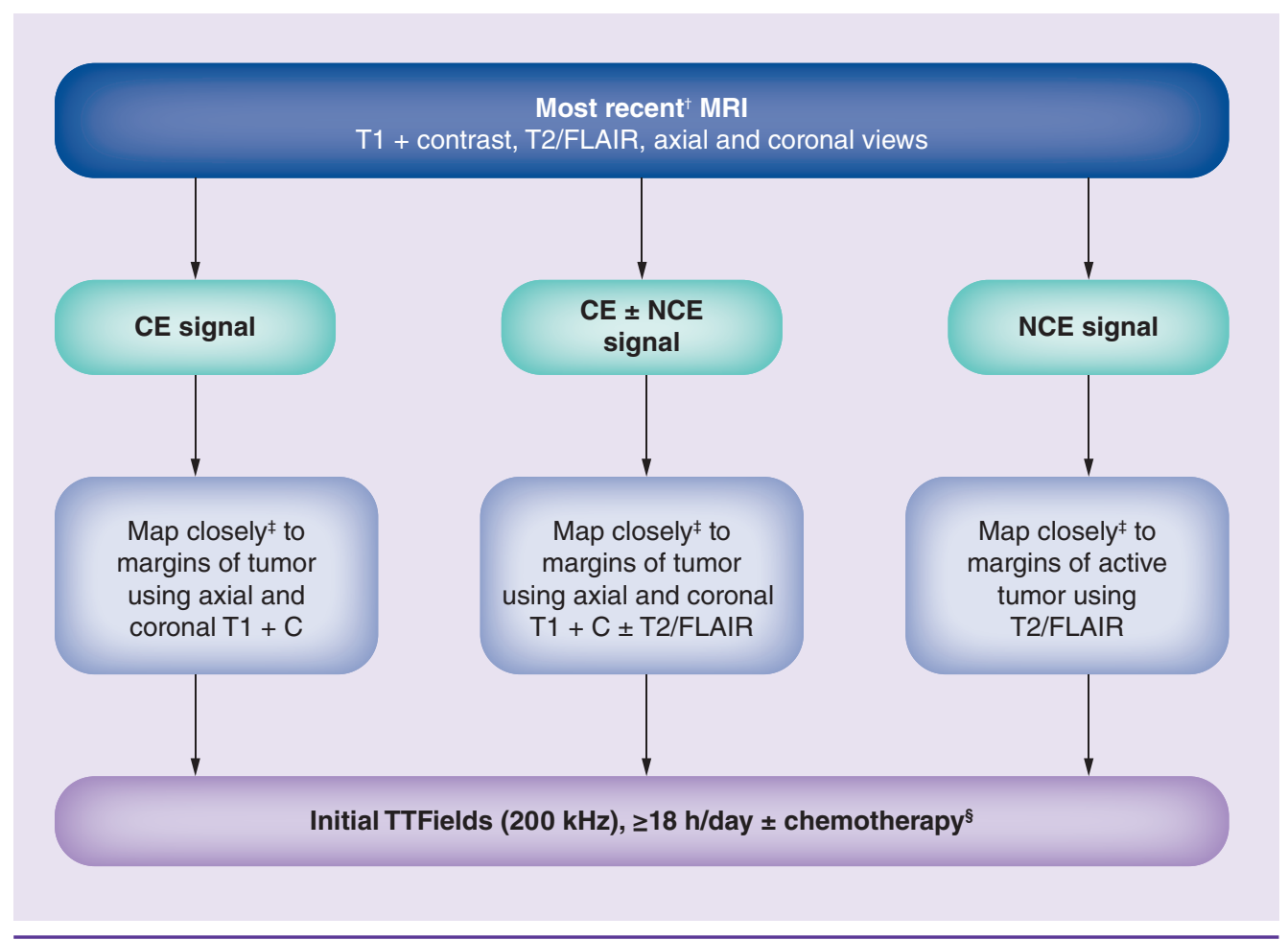

Figure 4. Tumor treating fields treatment planning algorithm for recurrent glioblastoma.

tWhere available, compare pretreatment and post-treatment imaging to determine areas of active disease versus post-treatment sequelae, especially in patients receiving antiangiogenic therapy.

${ }^{\ddagger}$ Coordinates for tumor location should closely approximate the edge of the region most representative of active tumor without including extra margin of normal tissue. This approach will maximize the field intensity in both the tumor volume and the proximal peritumoral brain zone.

sPatients initially receiving TTFields with maintenance TMZ who experience first progression, can remain on TTFields with a change to second-line chemotherapy.

C: Contrast; CE: Contrast enhancement; FLAIR: Fluid-attenuated inversion recovery; NCE: Noncontrast enhancement; TMZ: Temozolomide.

demonstrated that tumor regression and clinical benefit can still occur following initial early radiographic progression in patients receiving TTFields $[48,49]$. In a post hoc responder analysis from EF-11 (where TTFields were administered as monotherapy in recurrent GB patients), approximately a third of responders experienced progressive disease on their 2-month scan following initiation of TTFields. However, they subsequently developed delayed (median time to onset of objective response of 8 months), durable (a median duration of 7 months) and objective radiographic responses [48]. Similar observations are noted in patients receiving novel immunotherapeutics [50-52], which have recently prompted the Response Assessment in Neuro-Oncology Criteria (RANO) working group to develop an updated immunotherapy response assessment guideline (iRANO). This new guidance allows patients to remain on immune therapy if they demonstrate initial radiographic progression in the absence of significant clinical decline [53]. The iRANO updates recognize that immune therapies may take a longer period of time to confer a clinical benefit and facilitates a mechanism to prevent patients coming off therapy prematurely in the absence of true progressive disease, while ensuring patient safety. Similarly, TTFields require a longer period of treatment (at least 4 weeks of continuous exposure) in order to reach a state of tumor stabilization during the course of therapy [49]. As TTFields exert an antimitotic effect, there will be an initial period where the rate of tumor cell replication will exceed cell killing and clearance rates from the brain. Over time, the rate of cell cytotoxicity and clearance will eventually exceed replication rates and, therefore, there will be a delay until tumor shrinkage is observed on sequential 


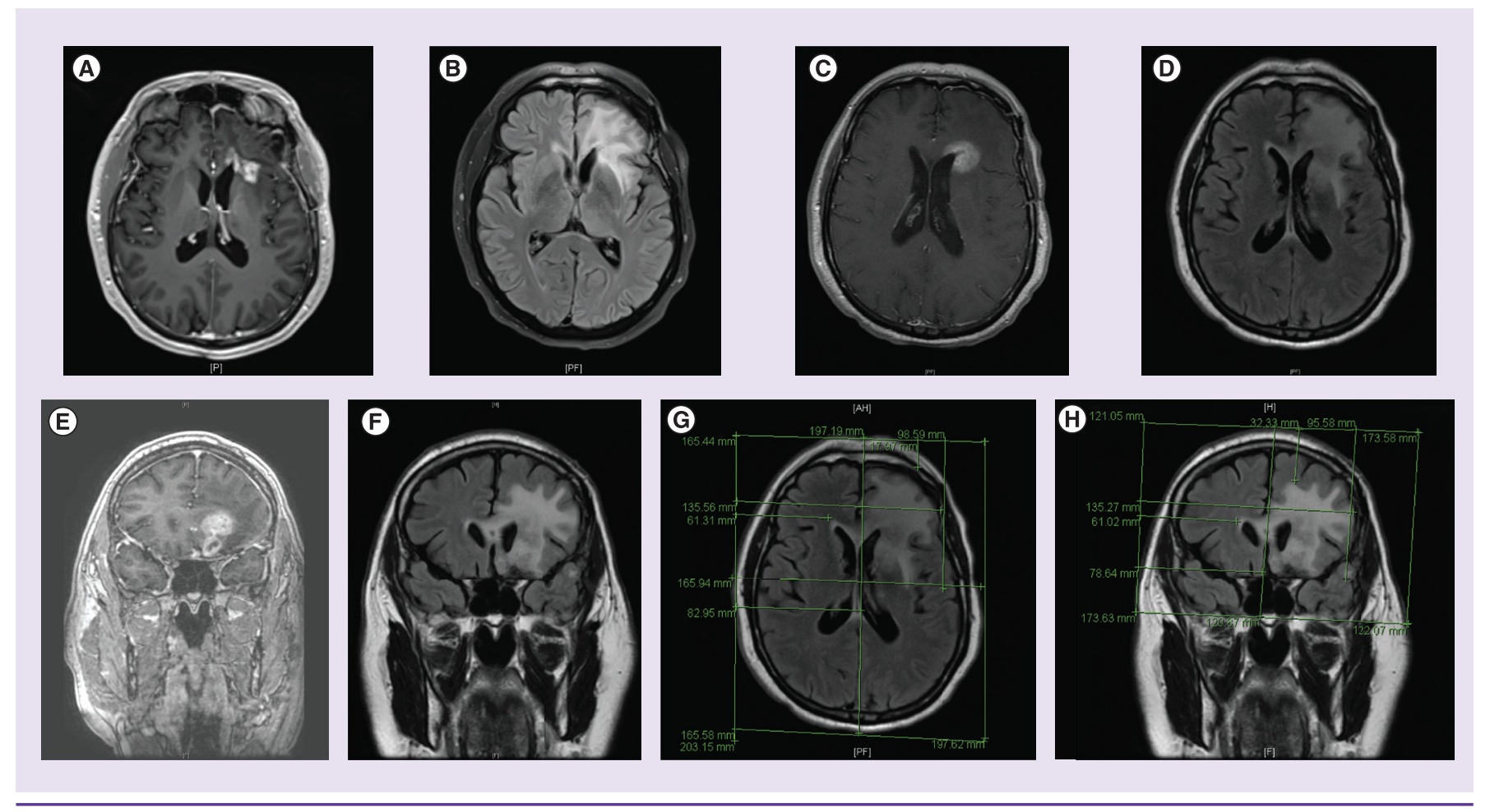

Figure 5. Treatment planning in a recurrent glioblastoma patient with nonmeasurable progression post-bevacizumab.

(A \& B) Preprogression axial T1 postcontrast and axial fluid-attenuated inversion recovery (FLAIR) abnormality, with no abnormality at the rostral, superior, lateral ventricle. (C-F) Postprogression axial T1 postcontrast and FLAIR abnormality, and coronal T1 postcontrast and FLAIR images. In this case, planning TTFields treatment using T1 postcontrast sequences will underestimate the extent of tumor progression. (G \& H) Treatment planning performed using axial and coronal FLAIR sequences, obtaining tumor coordinates to the margins of FLAIR abnormality.

imaging. In addition, early contrast-enhancing imaging changes may, in fact, represent a local inflammatory response to therapy, not necessarily overt tumor progression [54]. As such, this clinical guideline provides recommendations for maintaining and/or terminating therapy, based on a holistic assessment of the patient's clinical status, concurrent therapies and their imaging findings. Figure 7 describes the algorithm for response assessment and remapping patients receiving TTFields.

As TTFields do not impact the blood-brain barrier, any reduction in tumor size is indicative of a true anti-tumor effect (as opposed to pseudoresponse observed with antiangiogenic therapies). However, as TTFields may be used routinely in the clinical setting in conjunction with other chemotherapies, radiographic response assessment should be evaluated per the RANO criteria. In patients experiencing a complete or partial response, or those with stable disease, it is recommended to maintain TTFields therapy with the same array layout configuration. In instances where there has been a dramatic reduction in the extent of contrast enhancement ( $>50 \%$ reduction) in the absence of antiangiogenic therapy, it is also feasible for the treating physician to re-map the tumor based on the latest imaging, to ensure that the field is focused to the reduced area of contrast enhancement.

In patients exhibiting radiographic progression from the baseline planning scan, a number of factors should be considered. First, if the patient is a patient with newly diagnosed GB who has recently started TTFields in combination with maintenance TMZ, and has clinically deteriorated, it is recommended to continue TTFields and switch to a second-line chemotherapy as long as the patient remains compliant and able to tolerate the treatment. In the EF-14 Phase III study in patients with newly diagnosed GB, the median duration of TTFields therapy was 9 months, while median PFS was 7.1 months [25], with the majority of patients in the TTFields arm receiving secondline chemotherapies at first progression. If the patient has a recurrent $\mathrm{GB}$ and experienced 
significant neurologic decline consistent with the imaging changes since initiating TTFields, then treatment should be re-evaluated at the clinician's discretion.

In patients who exhibit radiographic changes consistent with disease progression at the 2-month scan, but who remain neurologically stable and are compliant with therapy, it is feasible to maintain them on TTFields therapy with the same transducer array layout and repeat the scan in another 2-3 months. If however, there appears to be a significant increase of $>25 \%$ in size, or new lesions have appeared distal to the original treated tumor bed at the first follow-up scan, then it is recommended to re-plan treatment to include the new or increased size lesions in the field and then continue with therapy. In general, any major changes in imaging from the baseline planning scan should be reviewed in the context of the TTFields treatment field, and re-planning should be considered at the treating physicians' discretion. Since TTFields exert
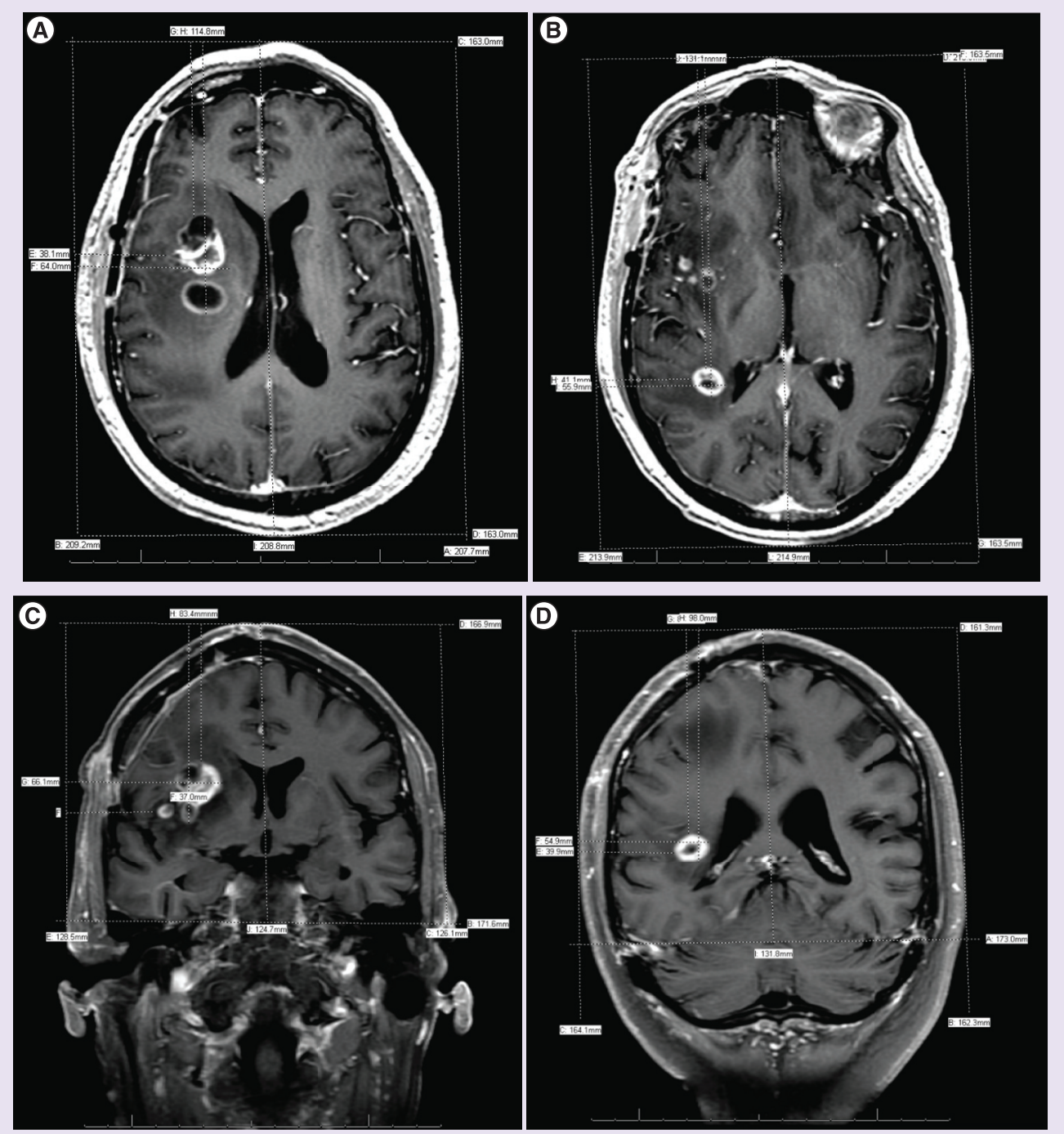

Figure 6. Treatment planning for multifocal tumors. (A \& B) Axial views of a multifocal tumor with a multicentric right frontoparietal lesion, and a smaller posterior lesion adjacent to the posterior horn of the lateral ventricle. (C \& D) Corresponding coronal views are shown. (B \& C) Treatment planning commences with a determination of head size on a slice at the apical level of the orbit and at the level of the ear canal per standard planning. Mutlifocal lesions are then mapped separately when $>1 \mathrm{~cm}$ apart. The multicentric anterior lesion is mapped as one contiguous lesion. 


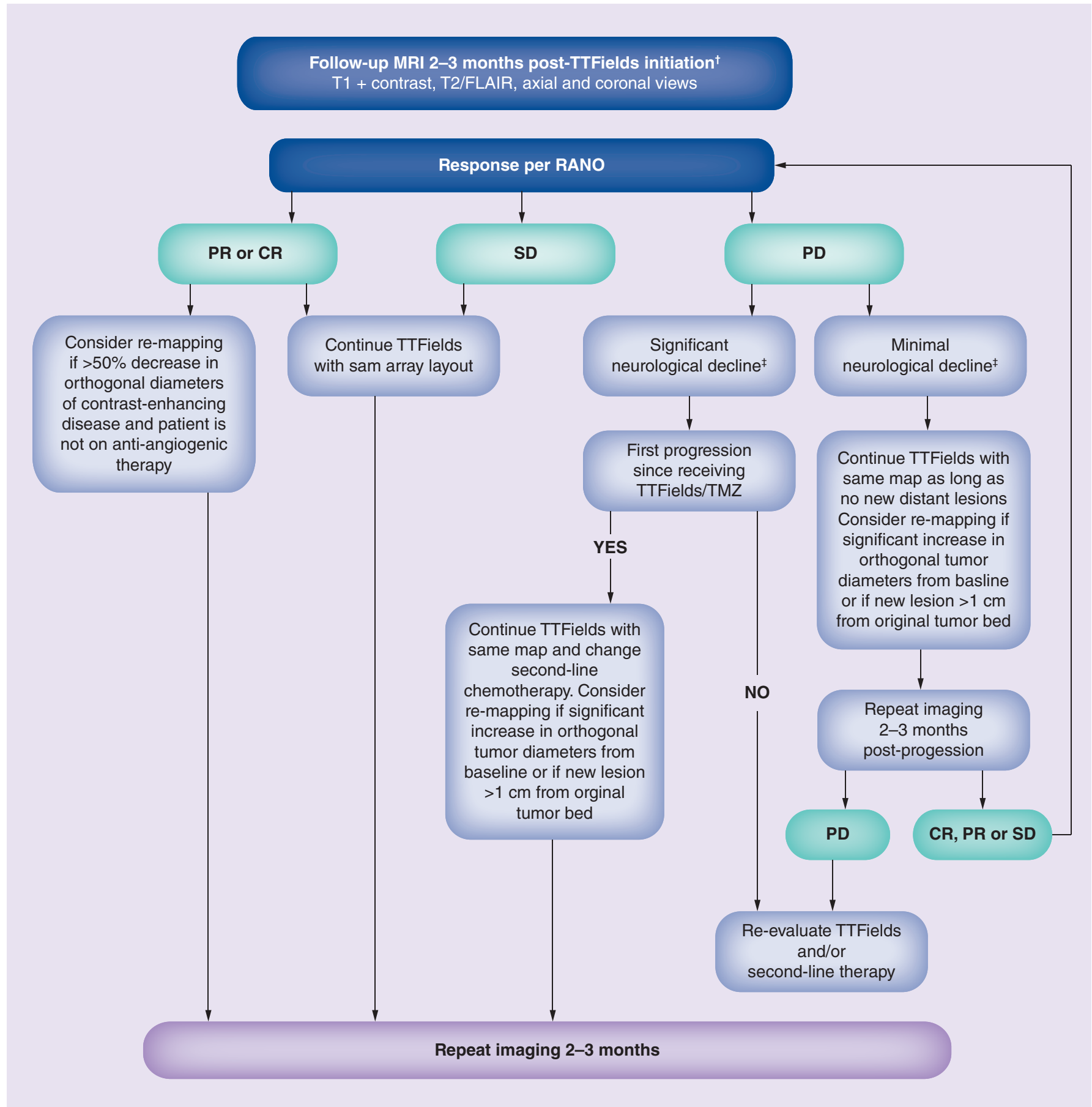

Figure 7. Response assessment and remapping algorithm for tumor treating fields in glioblastoma.

tServes as new reference scan if TTFields treatment continued.

${ }^{\ddagger}$ Clinical and neurological decline indicative of tumor progression and not attributable to a co-existing medical condition.

CR: Complete response; FLAIR: Fluid-attenuated inversion recovery; PD: Progressive disease; PR: Partial response; RANO: Response Assessment in Neuro-Oncology Criteria; SD: Stable disease; TMZ: Temozolomide; TTFields: Tumor treating fields.

an antimitotic effect exclusive to dividing cells, it may take several months of continuous use before true antitumor responses manifest on sequential imaging.

\section{Future perspective}

It has been more than a decade since the anticancer effects of TTFields were first characterized in GB cell lines and clinical studies were initiated. 
Today, TTFields are FDA-approved for newly diagnosed and recurrent GB and further development is underway in a variety of solid tumors [55-60]. Since TTFields are a comparatively new anticancer modality compared with surgery, chemotherapy and radiation therapy, this poses additional challenges in the clinic with regard to appropriately training physicians who are certified to plan treatment. This summary guideline proposes a framework for TTFields treatment planning that is consistent with current standards for MRI acquisition and response assessment, which are sensitive to the heterogeneity of tumor activity in imaging and which aim to maximize field intensity at the site of active tumor. Importantly, it proactively addresses early imaging changes that can occur in patients receiving TTFields which may not be indicative of disease progression. The algorithms provide clinicians a roadmap for preventing premature treatment discontinuation of a potentially beneficial modality while balancing safety. As experience with TTFields grows, this guideline will require future revisions to incorporate the integration of advanced imaging modalities in treatment planning, and the potential inclusion of radiologic biomarkers to assess response to treatment. The prospective inclusion of these guidelines in future clinical studies is warranted in order to establish their clinical effectiveness.

\section{Supplementary data}

To view the supplementary data that accompany this paper please visit the journal website at: http://www. futuremedicine.com/doi/full/10.2217/cns-2016-0032

\section{Acknowledgements}

The authors would like to thank A Chaudhry at Novocure, for medical writing support in the development of this manuscript

\section{Financial \& competing interests disclosure}

$J$ Battiste has disclosed serving as a consultant and speaker for Novocure, Inc. DA Bota has disclosed serving as a consultant and speaker for Novocure, Inc., and receiving funding from Triphase and travel, accommodation or other expenses reimbursement from ERC Belgium. $J$ Connelly has disclosed serving as a consultant for Novocure, Inc., and owning stock in Elli Lilly and Company, Halyard Health, Medtronic, AbbVie, Abbot Labs and Johnson and Johnson. D Damek has disclosed serving as a consultant for Novocure, Inc., and receiving funding from BMS, Celldex and AbbVie. F Iwamoto has disclosed serving as a consultant for Novocure, Inc., Regeneron, AbbVie and Prime Oncology, and receiving funding from Celldex, Northwest, Novocure, Inc. and BMS. N Mohile has disclosed serving as a consultant for Novocure, Inc. J Trusheim has disclosed serving as a consultant for Novocure, Inc., and owning stock in Fresca Medical. The authors have no other relevant affiliations or financial involvement with any organization or entity with a financial interest in or financial conflict with the subject matter or materials discussed in the manuscript apart from those disclosed.

\section{Open access}

This work is licensed under the Creative Commons Attribution-NonCommercial 4.0 Unported License. To view a copy of this license, visit http://creativecommons.org/ licenses/by-nc-nd/4.0/

\section{References}

1 Kirson ED, Gurvich Z, Schneiderman R et al. Disruption of cancer cell replication by alternating electric fields. Cancer Res. 64 , 3288-3295 (2004).

2 Lee SX, Wong ET, Swanson KD. Mitosis interference of cancer cells by NovoTTF-100A causes decreased cellular viability. Semin. Oncol. 14, iv9 (2012).

3 Lee SX, Wong ET, Swanson KD. Disruption of cell division within anaphase by tumor treating electric fields (TTFields) leads to immunogenic cell death. Neuro Oncol. 15(Suppl.), iii66-iii67 (2013).

4 Giladi M, Schneiderman RS, Voloshin T et al. Mitotic spindle disruption by alternating electric fields leads to improper chromosome segregation and mitotic catastrophe in cancer cells. Sci. Rep. 5, 18046 (2015).

5 Kirson ED, Dbaly V, Tovarys F et al. Alternating electric fields arrest cell proliferation in animal tumor models and human brain tumors. Proc. Natl Acad. Sci. USA 104, 10152-10157 (2007).

6 Novocure. Optune Instructions for use. Document number QSD-QR-330 (2015). http://html.investis.com

7 Lok E, Swanson KD, Wong ET. Tumor treating fields therapy device for glioblastoma: physics and clinical practice considerations. Expert Rev. Med. Devices 12(6), 717-726 (2015).

8 Gera N, Yang A, Holtzman TS, Lee SX, Wong ET, Swanson KD. Tumor treating fields perturb the localization of septins and cause aberrant mitotic exit. PLoS ONE 10, e0125269 (2015).

9 Gutin PH, Wong ET. Noninvasive application of alternating electric fields in glioblastoma: a fourth cancer treatment modality. In: American Society of Clinical Oncology Educational Book. Govindan R (Ed.). ASCO University, Alexandria, VA, USA, 126-131 (2012).

10 Yang A, Holtzman TS, Lee SX, Wong ET, Swanson KD. Alternating electric fields perturb the localization of cytokinetic furrow proteins and cause aberrant mitotic exit. Neuro Oncol. 16(Suppl. 5), v94 (2014).

11 Giladi M, Schneiderman RS, Porat Y et al. Mitotic disruption and reduced clonogenicity of pancreatic cancer cells in vitro and in vivo by tumor treating fields. Pancreatology 14, 54-63 (2014). 
12 Schneiderman R, Giladi M, Porat Y et al. TTFields reduce cancer cell clonogenic potential through abnormal chromosome segregation during mitosis. Cancer Res. 74(Suppl. 19), Abstract 5521 (2014).

13 Wong ET, Lok E, Gautam S, Swanson KD. Dexamethasone exerts profound immunologic interference on treatment efficacy for recurrent glioblastoma. Br. J. Cancer 113(2), 232-241 (2015).

14 Schneiderman RS, Voloshin-Sela T, Giladi M et al. $\mathrm{p} 53$ status dependence of tumor treating fields (TTFields) efficacy against glioma cells. Neuro Oncol. 17(Suppl. 5), v18-v40 (2015).

15 Porat Y, Shteingauz A, Giladi M et al. Alternating electric fields (TTFields) induce autophagy in human cancer cell lines. Presented at: 2016 Proceedings of the AACR. New Orleans, LA, USA, 16-20 April 2016 (Abstract 3543).

16 Schneiderman RS, Shteingauz A, Giladi M et al. Tumor treating fields (TTFields) reduce migration and invasion properties of human glioma cancer cells in vitro. Presented at: 2016 Proceedings of the AACR. New Orleans, LA, USA, 16-20 April 2016 (Abstract 5078).

17 Wenger C, Salvador R, Basser PJ, Miranda PC. The electric field distribution in the brain during TTFields therapy and its dependence on tissue dielectric properties and anatomy: a computational study. Phys. Med. Biol. 60 (18), 7339-7357 (2015).

18 Miranda PC, Mekonnen A, Salvador R, Basser PJ. Predicting the electric field distribution in the brain for the treatment of glioblastoma. Phys. Med. Biol. 59, 4137-4147 (2014).

19 Lok E, Hua V, Wong ET. Computed modeling of alternating electric fields therapy for recurrent glioblastoma. Cancer Med. 4(11), 1697-1699 (2015).

20 Wenger C, Salvador R, Basser PJ, Miranda PC. Improving Tumor Treating Fields treatment efficacy in patients with glioblastoma using personalized array layouts. Int. J. Radiat. Oncol. Biol. Phys. 94(5), 1137-1143 (2016).

21 Lemee JM, Clavreul A, Aubry M et al. Characterizing the peritumoral brain zone in glioblastoma: a multidisciplinary analysis. J. Neurooncol. 122(1), 53-61 (2015).

22 Lemee JM, Clavreul A, Menei P. Intratumoral heterogeneity in glioblastoma: don't forget the peritumoral brain zone. Neuro Oncol. 17(10), 1322-1332 (2015).

23 Chen L, Chaichana KL, Kleinberg L, Ye X, Quinones-Hinojosa A, Redmond K.

Glioblastoma recurrence patterns near neural stem cell regions. Radiother. Oncol. 116(2), 294-300 (2015).

24 Chaudhry A, Bomzon Z, Hershkovich $\mathrm{H}$, Garcia-Carracedo D, Wenger C, Palti Y. Tumor-treating fields (TTFields) intensity in the gross tumor volume and peritumoral brain zone: implications for local recurrence in glioblastoma [abstract]. Presented at: 107th Annual Meeting of the American Association for Cancer Research. New Orleans, LA, USA, 16-20 April 2016 (Abstract 2051).

25 Stupp R, Taillibert S, Kanner AA et al. Maintenance therapy with tumor-treating fields plus temozolomide vs temozolomide alone for glioblastoma: a randomized clinical trial. JAMA 314(23), 2535-2543 (2015).

26 Stupp R, Wong ET, Kanner AA et al. NovoTTF-100A versus physician's choice chemotherapy in recurrent glioblastoma: a randomised Phase III trial of a novel treatment modality. Eur. J. Cancer 48 , 2192-2202 (2012).

27 Zhu JJ, Pannullo S, Mehdorn M et al. Quality of life, cognitive function and functional status in the EF-14 Trial: a prospective, multi-center trial of tumor treating fields together with temozolomide compared to temozolomide alone in patients with newly diagnosed GBM. Neuro Oncol. 17(Suppl. 5), v9 (2015).

28 Kesari S, Ram Z, Investigators OBOTET. Tumor treating fields with chemotherapy compared to chemotherapy alone in glioblastoma patients at first recurrence: a post-hoc analysis of the EF-14 trial. Neuro Oncol. 17(Suppl. 5), v14 (2015).

29 Lacouture ME, Davis ME, Elzinga G et al. Characterization and management of dermatologic adverse events with the NovoTTF-100A System, a novel anti-mitotic electric field device for the treatment of recurrent glioblastoma. Semin. Oncol. 41(Suppl. 4), S1-S14 (2014).

30 Chaudhry A, Benson L, Varshaver M et al. NovoTTF-100A System (tumor treating fields) transducer array layout planning for glioblastoma: a NovoTAL system user study. World J. Surg. Oncol. 13, 316 (2015).

31 Chaudhry A, Benson L, Varshaver M, Farber O, Kirson E, Palti Y. Tumor treating fields (TTFields) transducer array layout planning for glioblastoma: results of a NovoTAL ${ }^{\mathrm{TM}}$ System user study. Neuro Oncol. 17(Suppl. 5), v10-v11 (2015).

32 Chaudhry A, Benson L, Varshaver M et al. NovoTTF ${ }^{\mathrm{TM}}-110 \mathrm{~A}$ System (tumor treating fields) transducer array layout planning for recurrent glioblastoma: results of a
NovoTAL ${ }^{\text {TM }}$ System User Study. Int. J. Radiat. Oncol. Biol. Phys. 93(No 3S), E50-E60 (2015).

33 Chaudhry A, Benson L, Varshaver $\mathrm{M}$ et al. NovoTTF-100A System (tumor treating fields) transducer array layout planning for glioblastoma: results of a NovoTAL system user study. J. Clin. Oncol. 33(Suppl. 15), e13029 (2015).

34 Gallego Perez-Larraya J, Lahutte M, Petrirena G et al. Response assessment in recurrent glioblastoma treated with irinotecan-bevacizumab: comparative analysis of the Macdonald, RECIST, RANO, and RECIST + F criteria. Neuro Oncol. 14(5), 667-673 (2012).

35 Elson A, Paulson E, Bovi J, Siker M, Schultz C, Laviolette PS. Evaluation of preradiotherapy apparent diffusion coefficient (ADC): patterns of recurrence and survival outcomes analysis in patients treated for glioblastoma multiforme. J. Neurooncol. 123(1), 179-188 (2015).

36 Nowosielski M, Wiestler B, Goebel G et al. Progression types after antiangiogenic therapy are related to outcome in recurrent glioblastoma. Neurology 82(19), 1684-1692 (2014).

37 Abdulla S, Saada J, Johnson G, Jefferies S, Ajithkumar T. Tumour progression or pseudoprogression? A review of posttreatment radiological appearances of glioblastoma. Clin. Radiol. 70(11), 1299-1312 (2015).

38 Melguizo-Gavilanes I, Bruner JM, GuhaThakurta N, Hess KR, Puduvalli VK. Characterization of pseudoprogression in patients with glioblastoma: is histology the gold standard? J. Neurooncol. 123(1), 141-150 (2015).

39 Suh CH, Kim HS, Choi YJ, Kim N, Kim SJ. Prediction of pseudoprogression in patients with glioblastomas using the initial and final area under the curves ratio derived from dynamic contrast-enhanced T1-weighted perfusion MR imaging. Am. J. Neuroradiol. 34(12), 2278-2286 (2013).

40 Galldiks N, Rapp M, Stoffels G et al. Response assessment of bevacizumab in patients with recurrent malignant glioma using $\left[{ }^{18} \mathrm{~F}\right]$ Fluoroethyl-L-tyrosine PET in comparison to MRI. Eur. J. Nucl. Med. Mol. Imaging 40 (1), 22-33 (2013).

41 Choi YJ, Kim HS, Jahng GH, Kim SJ, Suh DC. Pseudoprogression in patients with glioblastoma: added value of arterial spin labeling to dynamic susceptibility contrast perfusion MR imaging. Acta Radiol. 54(4), 448-454 (2013). 
42 Brandsma D, Stalpers L, Taal W, Sminia P, Van Den Bent MJ. Clinical features, mechanisms, and management of pseudoprogression in malignant gliomas. Lancet Oncol. 9(5), 453-461 (2008).

43 Huang RY, Neagu MR, Reardon DA, Wen PY. Pitfalls in the neuroimaging of glioblastoma in the era of antiangiogenic and immuno/targeted therapy - detecting illusive disease, defining response. Front. Neurol. 6, 33 (2015).

44 Hygino Da Cruz LC Jr, Rodriguez I, Domingues RC, Gasparetto EL, Sorensen AG. Pseudoprogression and pseudoresponse: imaging challenges in the assessment of posttreatment glioma. Am. J. Neuroradiol. 32(11), 1978-1985 (2011).

45 Prager AJ, Martinez N, Beal K, Omuro A Zhang Z, Young RJ. Diffusion and perfusion MRI to differentiate treatment-related changes including pseudoprogression from recurrent tumors in high-grade gliomas with histopathologic evidence. Am. J. Neuroradiol. 36(5), 877-885 (2015).

Chu HH, Choi SH, Ryoo I et al.

Differentiation of true progression from pseudoprogression in glioblastoma treated with radiation therapy and concomitant temozolomide: comparison study of standard and high-b-value diffusionweighted imaging. Radiology 269(3), 831-840 (2013).

Thomas RP, Xu LW, Lober RM, Li G, Nagpal S. The incidence and significance of multiple lesions in glioblastoma. J. Neurooncol. 112(1), 91-97 (2013).

Wong ET, Lok E, Swanson KD et al. Response assessment of NovoTTF-100A versus best physician's choice chemotherapy in recurrent glioblastoma. Cancer Med. 3 , 592-602 (2014).

49 Vymazal J, Wong ET. Response patterns of recurrent glioblastomas treated with tumor-treating fields. Semin. Oncol. 41(Suppl. 6), S14-S24 (2014).

50 Hamid O, Robert C, Daud A et al. Safety and tumor responses with lambrolizumab (anti-PD-1) in melanoma. N. Engl. J. Med. 369(2), 134-144 (2013).

51 Okada H, Pollack IF. Do we need novel radiologic response criteria for brain tumor immunotherapy? Expert Rev. Neurother. 11(5), 619-622 (2011).

52 Topalian SL, Sznol M, Mcdermott DF et al. Survival, durable tumor remission, and long-term safety in patients with advanced melanoma receiving nivolumab. J. Clin. Oncol. 32(10), 1020-1030 (2014).

53 Okada H, Weller M, Huang R et al. Immunotherapy response assessment in neuro-oncology: a report of the RANO working group. Lancet Oncol. 16(15), e534-e542 (2015).

54 New P, Powell S. Pathology of cases of imaging progression in patients with glioblastoma who have been treated with the novocure - TTF device in the EF-14 trial. Neuro Oncol. 16(Suppl. 5), v164 (2014).
55 Brozova H, Lucas A, Salmaggi A, Vymazal J. COMET: a Phase II randomized study of TTFields versus supportive care in non-smallcell lung cancer patients with $1-5$ brain matastases - initial safety results. Neuro Oncol. 17(Suppl. 5), V-46 (2015).

56 Kirson ED, Giladi M, Gurvich Z et al. Alternating electric fields (TTFields) inhibit metastatic spread of solid tumors to the lungs. Clin. Exp. Metastasis 26, 633-640 (2009).

57 Rivera F, Gallego J, Guillen Ponce C, Benavides M, Lopez-Martin JA, Küng M. PANOVA: a pilot study of TTFields concomitant with gemcitabine for front-line therapy of advanced pancreatic adenocarcinoma. J. Clin. Oncol. 33(Suppl.), Abstract 269 (2015)

58 Pless M, Droege C, Von Moos R, Salzberg M, Betticher D. A Phase I/II trial of tumor treating fields (TTFields) therapy in combination with pemetrexed for advanced non-small-cell lung cancer. Lung Cancer 81, 445-450 (2013).

59 Weinberg U, Farber O. A Phase II trial of TTFields with chemotherapy for first line treatment of malignant mesothelioma. Presented at: IASLC World Congress on Lung Cancer. Denver, CO, USA, 6-9 September 2015.

60 Mehta MP, Gondi V, Brown PD. A Phase III study of radiosurgery with TTFields for 1-10 brain metastases from NSCLC. Sub to IASLC World Congress on Lung Cancer. Denver, CO, USA, 6-9 September 2015. 